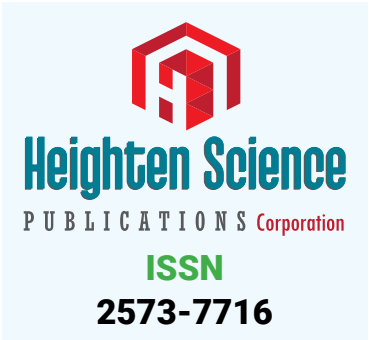

*Address for Correspondence: Sannia Mia Sveningsen Sjostedt, Department of Otorhinolaryngology and Audiology, Rigshospitalet, Blegdamsvej 9B, F2071, Copenhagen, Denmark, Email: sannia@dadlnet.dk

Submitted: 11 September 2017

Approved: 18 September 2017

Published: 20 September 2017

Copyright: @ 2017 Sjostedt SMS, et al. This is an open access article distributed under the Creative Commons Attribution License, which permits unrestricted use, distribution, and reproduction in any medium, provided the original work is properly cited.
Case Report

\section{Kikuchi's disease in a young Scandinavian woman}

\author{
Sannia Mia Sveningsen Sjostedt ${ }^{1 *}$, Eva Hebbelstrup Rye \\ Rasmussen ${ }^{2}$, Anette Bygum ${ }^{3}$ and Line Kanstrup Springborg ${ }^{4}$ \\ 'Department of Otorhinolaryngology and Audiology, Rigshospitalet, Blegdamsvej 9B, F2071, \\ Copenhagen, Denmark \\ 2Department of Otorhinolaryngology and Audiology, Rigshospitalet, Blegdamsvej 9B, F2071, \\ Copenhagen, Denmark \\ ${ }^{3}$ Professor, Department of Dermatology and Allergy, Odense Hospital, Odense, Denmark \\ ${ }^{4}$ Consultant, Department of Otorhinolaryngology and Audiology, Rigshospitalet, Blegdamsvej \\ 9B, F2071, Copenhagen, Denmark
}

\section{Abstract}

A 21 year-old woman presented with cervical lymphadenopathy and night sweats. Initially, lymphoma was suspected and one of the enlarged lymph nodes was excised in order to make a diagnosis. Histopathological examination revealed Kikuchi's disease, which is usually a benign syndrome with spontaneous resolution. The disease is very rare, particularly in Caucasian populations. A higher incidence is seen in patients of Asian descent.

The patient had persistent swelling of cervical lymph nodes over several months and further lymph nodes were removed as malignant transformation was suspected. However examinations showed only Kikuchi's disease. There was given no specific treatment. Follow-up appointments were performed biannually and the patient is currently awaiting removal of yet another swollen lymph node on suspicion of relapse of her Kikuchi's disease.

\section{Introduction}

Kikuchi's disease (also known as Kikuchi-Fujimoto's disease or histiocytic necrotizing lymphadenitis) is a rare differential diagnosis to be considered when encountering a patient with cervical lymphadenopathy. It was first described in Japan in 1972 and is still most common in Asian populations [1-3], but has also been described in Caucasian patients [1,4]. For unknown reasons three to four times more women than men acquire the disease [1].

Onset of the disease is most often before the fourth decade. General symptoms are usually vague (leucopenia, fatigue, low-grade fever, lymphadenitis) and might be confused with several other diagnoses i.e. malignant disease, infections or autoimmune disease.

Usually, the disease presents as painful lymphadenopathy combined with fever, malaise and fatigue. Most commonly, the cervical lymph nodes are involved (56-98 \%), measuring $0.5-4 \mathrm{~cm}$ in diameter. Other regions of lymph nodes can be involved, such as the supraclavicular, axillary, superior mediastinal, portacaval or retroperitonal. The disease can also present as fever of unknown origin as the sole symptom. Symptoms often have acute or sub-acute onset. The unspecific symptoms and the rarity of the illness means that there are many other differential diagnoses to consider, e.g. lymphoma, tuberculosis, metastatic disease, infectious mononucleosis, systemic lupus erythematous (SLE) or cat scratch disease [1,5-8].

How to cite this article: Sjostedt SMS, Rasmussen EHR, Bygum A, Springborg LK. Kikuchi's disease in a young Scandinavian woman. Heighpubs Otolaryngol and Rhinol. 2017; 1: 069-076. https://doi.org/10.29328/journal.hor.10010012 
No specific cause for Kikuchi's disease has been verified, but an association to a viral infection and a subsequent immunological response has been proposed. However, a few cases of fatal Kikuchi-like disease have been described, some cases in patients already suffering from a connective tissues disease $[2,4]$.

Fine-needle aspiration biopsy (FNAB) cannot provide the diagnosis, so a whole lymph node should be excised for histopathological examination [9]. The typical findings are coagulative necrosis, foci of various histiocytes, debris, immunoblasts (T-cells) together with large and small lymphocytes $[9,10]$.

Blood samples usually show leucopenia with lymphocytosis and atypical lymphocytes in peripheral blood, erythrocyte sedimentation rate (ESR) and transaminases as well as lactate dehydrogenase (LDH) are often elevated [4].

Kicuchi's disease is a histolopathologic diagnosis based on focal cortical and paracortical necrosis with marked karyorrhexis and an infiltrate of crescentic histiocytes and plasmacytoid monocytes without neutrophils in lymph nodes. Cellular infiltrates consist of CD68+/CD123+ plasmacytoid histiocytes and transformed lymphocytes, mainly CD8+ T-lymphocytes expressing perforin involved in provoking apoptosis in target cells. Tubuloreticular structures in histiocytes, activated $\mathrm{T}$ cells and endothelial cells resemble those seen in SLE and viral disease [5]. However in SLE lymphadenitis neutrophils, plasma cells and haematoxylin bodies are often present [2].

The etiology of the disease remains unknown, but a hyperimmune reaction has been proposed. It is believed that an unknown infectious trigger incites an immune response of T-lymphocytes and histiocytes in which the cellular destruction seen is apoptosis brought on by CD8 T-lymphocytes. One study showed an oligoclonal pattern of T-cell receptor antigen rearrangement, thus indicating a benign reaction rather than clonal T-cell malignancy [5]. A variety of infectious agents (Brucella, Yersenia, toxoplasmosis, Ebstein-Barr virus (EBV), human herpes virus 6 (HHV6), hepatitis B virus (HBV), cytomegalovirus (CMV)) are suspected, but blood cultures, polymerase chain reaction (PCR), serological studies and hydridization technique examinations are usually negative $[1,2,4]$. Cutaneous involvement is seen in approximately $30 \%$ of patients [3].

The disease is usually benign, self-limiting and requires no specific treatment. Some recommend non-steroidal anti-inflammatory drugs (NSAID) for symptom relief and in more aggressive cases systemic corticosteroids $[5,6,11]$. Symptoms most frequently resolve within 6 months [1,9], but has been reported to last up to 37 months [12].

Recurrence is seen in only 3-4\% of patients [1], but may appear with as much as 19 years delay $[4,5]$. Several papers recommend long term follow-up because of an assumed connection with SLE $[2,5]$.

\section{Case Presentation}

We present a case of Kikuchi's disease in a young woman of northern European descent. In May of 2015, a 21 year-old woman presented to the Ear-, Nose-, and Throat (ENT) department with left-sided, tender cervical lymphadenopathy during approximately four to five months. The swelling was accompanied by frequent and severe night sweats, but no weight loss, fever or other B-symptoms (fatigue, decreased appetite) were encountered. The swelling on the neck had started during a common cold episode, but contrary to the symptoms of the upper airway infection, it did not resolve.

The patient was referred to the ENT department in a fast-track cancer referral program from her general practitioner who suspected lymphoma. She was otherwise healthy with no regular use of medication, except oral contraceptives. There was no family history nor did she smoke or report any alcohol consumption. Her BMI was 30.1 . 
When first evaluated, the patient had enlarged lymph nodes bilaterally in close relation to the sternocleidomastoid muscle (level 2,3), as well as a collection of enlarged tender lymph nodes on the left side of the neck (level 5). Ultrasound examination showed small normal looking lymph nodes along the vessels of the neck bilaterally. Several larger lymph nodes were seen laterally on the neck; on the left side as a conglomerate of enlarged lymph nodes was found. The largest measured $10 \times 10 \times 10$ $\mathrm{mm}$.

The rest of the oto-rhino-laryngological examination, including a fiberoptic rhinopharyngo-laryngoscopy, was normal. All the blood samples taken were normal, including Complete Blood Count (CBC) and Lactate Dehydrogenase (LDH) (see investigations).

A FNAB was performed from the two largest lymph nodes and sent for rapid analysis. The examination showed normal lymphocytic population. However as lymphoma could not be ruled out with FNAB alone, one of the enlarged lymph nodes was removed surgically less than five days of her first appointment. The histologic examination revealed Kikuchi's disease and no treatment was initiated.

The patient came back after three months with another enlarged lymph node located underneath the original scar measuring $20 \times 20 \times 10 \mathrm{~mm}$ on ultrasound examination. A FNAB was taken from this lymph node; the histology examination showed a normal lymphocytic population.

The patient was then referred to the department of infectious medicine for evaluation. New blood samples were taken; the patient was HIV-negative, toxoplasmosis negative, CMV negative but showed signs of previous EBV-infection.

Eight months after the initial contact, the patient was re-referred to the ENT department with increasing swelling of the cervical lymph nodes and persistent night sweats. The infectious medicine specialist was concerned if patient had developed lymphoma unrelated to Kikuchi's disease.

An ultrasound examination showed two enlarged lymph nodes near the mandibular angle and a collection of six enlarged lymph nodes near the midline of the neck, the

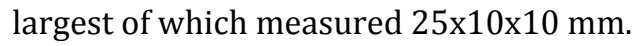

Three additional lymph nodes including the two enlarged ones visualized on ultrasound examination were excised. Histopathological examination again showed Kikuchi's disease. The patient had follow-up visits at the department of infectious disease biannually and is now awaiting removal of yet another swollen cervical lymph node on suspicion of relapse of her Kikuchi's disease.

\section{Blood test results}

- Hgb $8.4 \mathrm{mmol} / \mathrm{L}$ [normal range 7.3-9.5 mmol/L]

- Leucocytes $7.8 \times 10^{9} / \mathrm{L}$ [normal range $\left.3.5-8.8 \times 10^{9} / \mathrm{L}\right]$

- B-basofilocytes $0,03 \times 10^{9} / \mathrm{L}$ [normal range $0.00-0.10 \times 10^{9} / \mathrm{L}$ ]

- B-eosinofilocytes $0,07 \times 10^{9} / \mathrm{L}$ [normal range $0.04-0.50 \times 10^{9} / \mathrm{L}$ ]

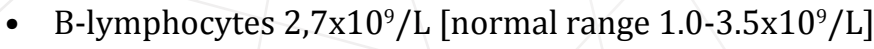

- B-metamyelo/myelo/promyelocytes $0,01 \times 10^{9} / \mathrm{L}$ [normal range $0.00-0.04 \times 10^{9} / \mathrm{L}$ ]

- B-monocytes $0.6 \times 10^{9} / \mathrm{L}$ [normal range $0.20-0.76 \times 10^{9} / \mathrm{L}$ ]

- B-neutrocytes $4.4 \times 10^{9} / \mathrm{L}$ [normal range $1.6-5.9 \times 10^{9} / \mathrm{L}$ ] 
- B-trombocytes $422 \times 10^{9} / \mathrm{L}$ [normal range $145-390 \times 10^{9} / \mathrm{L}$ ]

- Sedimentary reaction: 4 working units (2-20) (2/10 2015)

- $\quad$ CRP $4 \mathrm{mg} / \mathrm{L}$ [normal range $<10 \mathrm{mg} / \mathrm{L}$ ]

- Basic phosphatase $58 \mathrm{U} / \mathrm{L}$ [normal range 35-105 U/L]

- Lactate dehydrogenase $167 \mathrm{U} / \mathrm{L}$ [normal range 105-205 U/L]

- Aspartattransaminasis $34 \mathrm{U} / \mathrm{L}$ [normal range 15-35 U/L]

- P-humane immunodeficiency virus $1+2$ (antibody+Ag) 0 [normal range 0-0]

- M. tuberculosis quantiferon negative

- EBV AB IgM (VCA) negative

- EBNV AB IgG positive

- EBV IgG(VCA) positive

- Toxoplasmosis antibody IgM and IgG negative

- CMV antibody IgM and IgG negative

Histopathological examination of excised lymph node: October 2015, cervical lymph node, level $V$ sinister: enlarged lymph node with preserved architecture, cortical B-cell follicles, interfollicularly dominated by $\mathrm{T}$ cells without atypical features. Larger necrotic areas are seen, containing cellular debris and fibrinoid deposits, no vital granulocytes. Corresponding to necrotic areas collections of histiocytic cells partly MPO-positive, no Hodgkin-cells, no suspicion of malignant lymphoma. No epithelial cells. No fungi or acid fast bacteria (Figures 1-4).

\section{Treatment}

The patient was treated conservatively as appropriate, since no specific treatment is available.

\section{Outcome and follow-up}

The patient awaits removal of a new enlarged cervical lymph node, another relapse is suspected.

\section{Discussion}

The patient presented with persistently enlarged cervical lymph nodes and non-

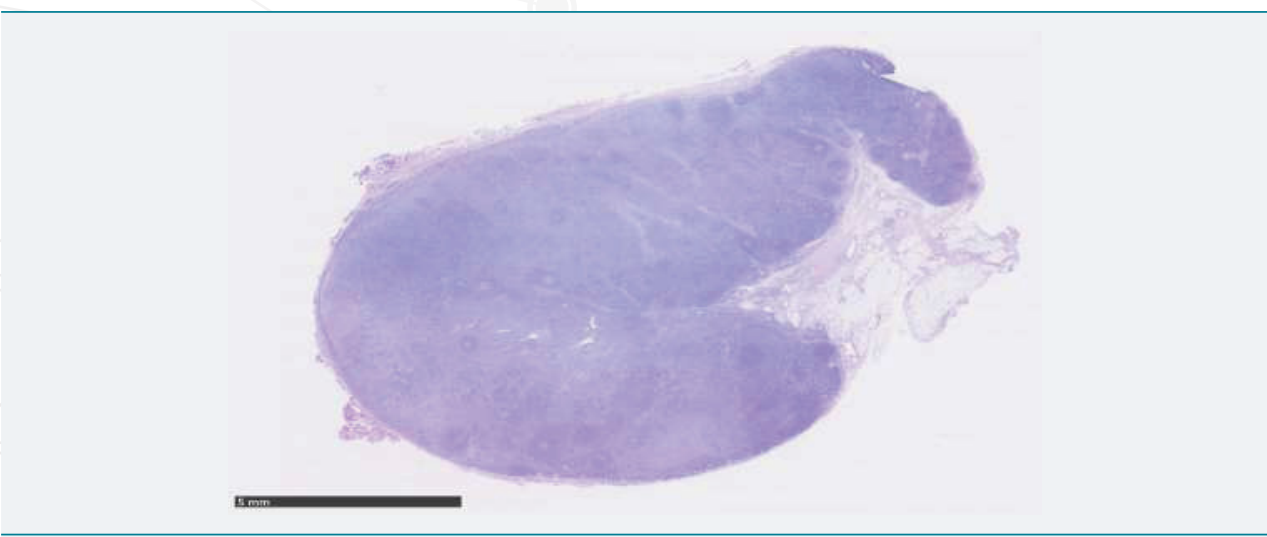

Figure 1: Lymphnode with follicles and necrotic areas with collections of histiocytic cells partially myeloperoxidase positive. No Hodgkin cells. 


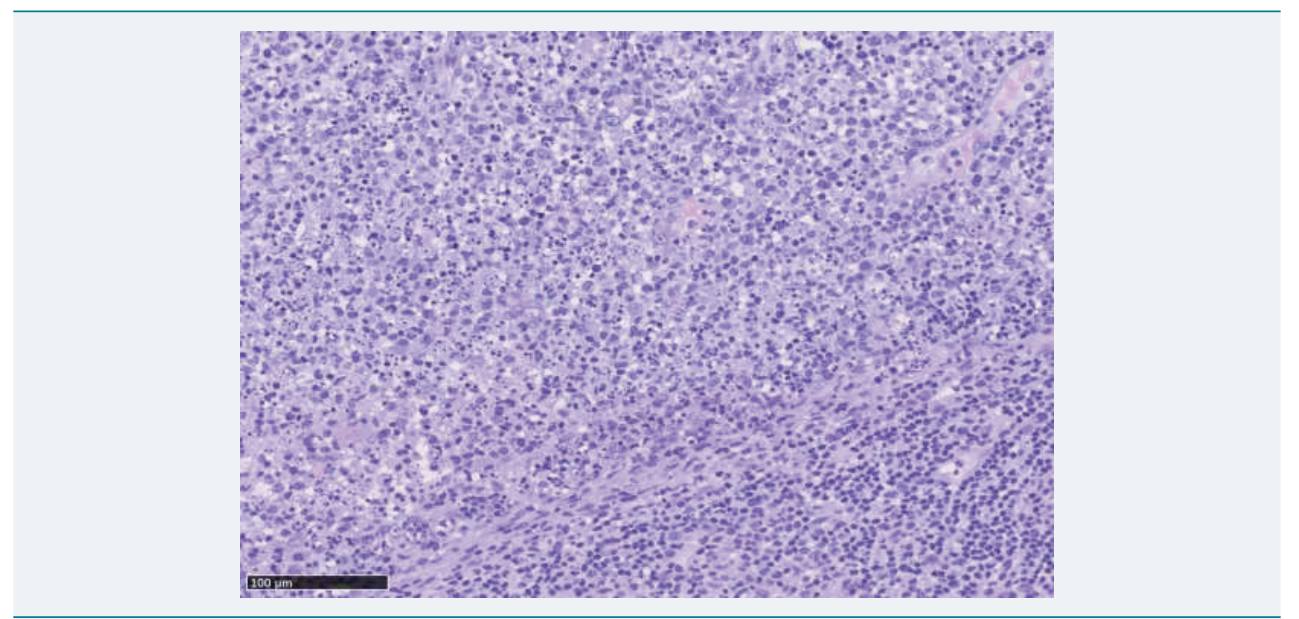

Figure 2: Magnification of Figure 1.

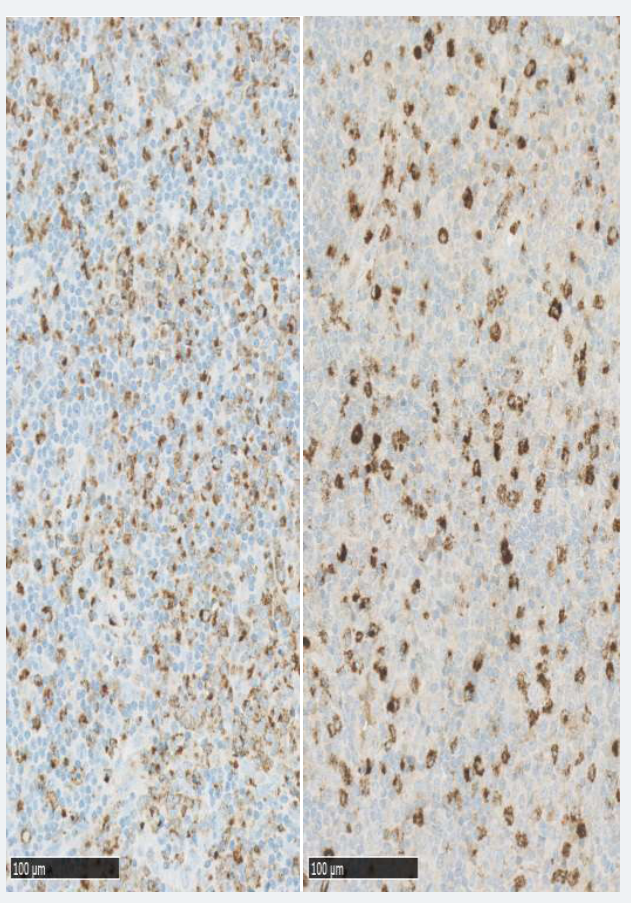

Figure 3: Showing CD68 (a) and MPO (b) staining.

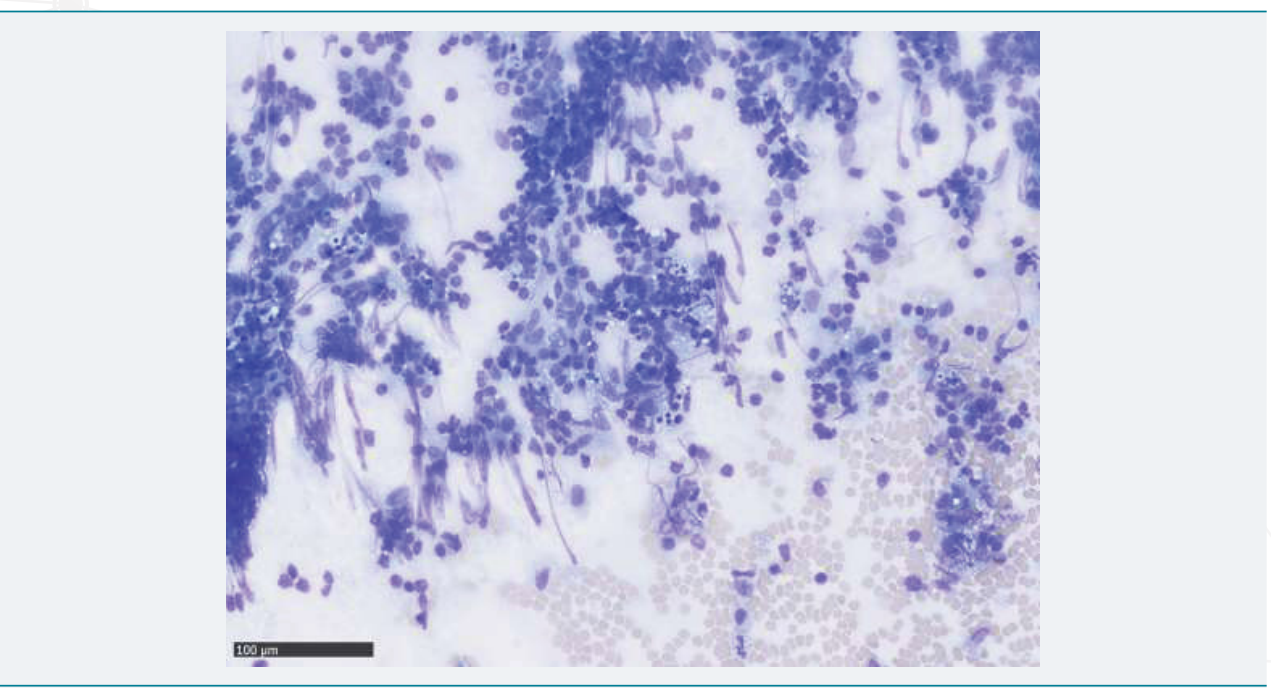

Figure 4: Fine Needle Aspiration biopsy showing a mixed population of lymphocytic cells. 
specific general symptoms. A fine needle aspirate (FNA) showed normal lymhocytic population. A lymph nodes was removed in order to procure a diagnosis. The procedure was done five days after the patient's first appointment, as lymphoma could not be ruled out. The patient was re-referred from the department of infectious medicine, where she was out-patient, under suspicion of lymphoma after 8 months. An FNA from this also showed normal lymphocytic population and this lymph node too was removed. Both histopathological examinations showed Kikuchi's disease.

Kikuchi's disease has been mistaken for malignant lymphoma and some initially misdiagnosed patients were treated with chemotherapy $[5,9]$. It is thus important to be aware of this entity.

The histopathological diagnosis can be elusive and Kikuchi's disease has been mistaken for reactive lymphadenitis as well as NHL [9]. The latter perhaps due to obliteration of sinuses and proliferations of plasmacytoid monocytes and immunoblasts [10]. Other histological, as well as clinical differential diagnosis include tuberculous adenitis, cat scratch disease and herpes virus infection [9].

Depending on the site of the enlarged lymph node, Kikuchi's disease can give rise to focal as well as universal symptoms, such as brachial plexus neuritis (supraclavicular region) [8], gastrointestinal symptoms (periportal and retroperitoneal lymph nodes [6].

There are reports of co-occurrence of Kikuchi's disease and other diseases, such as Still's disease, where a higher proportion of patients have rashes and arthritis [7] and thyroid cancer, where Kikuchi's disease mimicked a metastasis [1].

Our case is unusual because the patient had two relapses, which is rare [10], months after first having a lymph node removed for diagnostic purposes. The relapses occurred at the same anatomical site and the first lymph nodes were surgically removed in order to exclude lymphoma. The patient awaits removal of the lymph node with the latest suspected relapse.

A connection between Kikuchi's disease and SLE is suspected. The two diseases share a preponderance among younger women and have similarities at the ultrastructural level by microscopy $[2,4,9]$. Skin lesions may indicate an evolution into SLE. An association with other connective tissue diseases such as Still's disease, Hashimoto's thyroiditis, polymyositis, autoimmune hepatitis, Sjögren's syndrome, cutaneous necrotizing vasculitis, hemophagocytic lymphohistiocytosis, antiphospholipid syndrome, as well as mixed connective tissue disease is also suspected $[5,7,10]$.

Differential diagnosis to be considered with enlarged lymph nodes are lymphoma (malignant disease, often generalized lymphadenopathy, typically associated with abnormal blood samples, hepatosplenomegaly or B-symptoms [13], Human Immunodeficiency Virus (HIV) (infection with HIV. A benign lymphadenopathy can be seen in patients with HIV. This is, however, also associated with Hodgkin's disease (HD), non-Hodgkin's lymphoma (NHL), Castleman's disease (CD) and Kaposi's sarcoma (KS), thus making a lymph node biopsy mandatory, even if HIV-infection is known [13], Cat scratch disease (infection with Bartonella henselae found in cats and their fleas, contagious from bites and scrathes. The disease is most common in children, and presents as regional lymphadenopathy with malaise, aches, and in some cases lowgrade fewer. The infection is usually self-limiting, though spread to eyes, liver, spleen or CNS may warrant the use of antibiotics(Klotz et al. 2011)) [14], Cytomegalovirus (CMV)/Ebstein-Bar Virus (EBV) (infection with EBV and CVM. Can present persistent, multifocal lymphadenopathy. EBV is associated with lymphoproliferative disease such as infectious mononucleosis and HD as well as NHL, particularly in the immunocompromised patient [13], Toxoplasmosis (infection with the intracellular 
parasite; Toxoplasmosa Gondii, from undercooked meat contaiting cysts or water/ food contaminated with oocyst from cat faeces. The infection is usually asymptomatic, but can present as isolated non-tender discrete lymphadenopathy of the neck. Most cases are benign, requiring no antibiotics [15], Castleman's disease (An atypical lymphoproliferative disorder with malignant potential. The disease is divided into two groups based on clinical findings: unicentric and multicentric Castleman's disease, the first of which is often asymptomatic, most commonly present in the mediastinum, usually an incidental finding on imaging, the latter a systemic disease with significant peripheral lymphadenopathy and hepatosplenomegaly, associated with HIV and KS, rapidly progressive fatal form and a more chronic form [13], and Lymphomatoid granulomatosis (EBV-related B-cell lymphoproliferative disease, originally described as pulmonary angiitis with infiltrates of atypical B cells infected with EBV. The disease usually presents in patients between 30 and 50 years of age with shortness of breath, cough and chest pains as well as systemic symptoms such as fatigue, fever and weight loss. 20-50 \% of patients have skin lesions (erythematous rash, subcutaneous nodules, or plaques). The clinical course varies from spontaneous resolution to rapid fatality [13].

\section{Learning points/take home messages}

Kikuchi's disease is a rare differential diagnosis in a patient with enlarged lymph nodes and/or fever of unknown origin

- The diagnosis is based on histopathological exam

No specific treatment is required, the condition in most cases runs a benign course, and symptoms remit within 6 months

A possible association with systemic lupus erythematosus makes long-term follow-up a prudent choice.

\section{References}

1. Garg S, Villa M, Asirvatham JR, Mathew T, Auguste LJ. Kikuchi-Fujimoto Disease Masquerading as Metastatic Papillary Carcinoma of the Thyroid. Int J Angiol. 2015; 24: 145-150. Ref.: https://goo.gl/oTUvqw

2. Sharma V, Rankin R. Fatal Kikuchi-like lymphadenitis associated with connective tissue disease: a report of two cases and review of the literature. Springerplus. 2015; 8: 167. Ref.: https://goo.gl/jZJGo7

3. Deaver D, Naghashpour M, Sokol L. Kikuchi-fujimoto disease in the Unites States: Three case reports and review of the literature. Mediterr J Hematol Infect Dis. 2014; 6. Ref.: https://goo.gl/rXMDdB

4. Payne JH, Evans M, Gerrard MP. Kikuchi-Fujimoto disease: a rare but important cause of lymphadenopathy. Acta paediatr. 2003; 92: 261-264. Ref.: https://goo.gl/3YVpwK

5. Mrówka-Kata K, Kata D, Kyrcz-Krzemień S, Helbig G. Kikuchi-Fujimoto and Kimura diseases: The selected, rare causes of neck lymphadenopathy. Eur Arch Otorhinolaryngol. 2010; 267: 5-11. Ref.: https://goo.gl/tCwM52

6. Vu D, Reddy S, Day L, Aydin N, Misra S. Kikuchi-Fujimoto disease: Unusual presentation of rare disease. Clin Pract. 2015; 6: 16-17. Ref.: https://goo.gl/ZdM2Ro

7. Toribio KA, Kamino H, Hu S, Pomeranz M, Pillinger MH. Co-occurrence of Kikuchi-Fujimoto's disease and Still's disease: case report and review of previously reported cases. Clin Rheumatol. 2015; 34: 2147-2153. Ref.: https://goo.gl/cYxTZ7

8. Gupta R, Kayal A, Rajawat V. Kikuchi-Fujimoto disease presenting as brachial plexus neuritis: A case report. Indian J Neurosurg. 2016; 5: 110-113. Ref.: https://goo.gl/PZqnjy

9. Sopeña B, Rivera A, Caritina VT, Fluiters E, Joaquin GC. Autoimmune manifestations of kikuchi disease. Seminars in Arthritis and Rheumatism. 2012; 41: 900-906. Ref.: https://goo.gl/2W5pRA

10. Seong GM, Kim JH, Lim GC, Kim J. Clinicopathological review of immunohistochemically defined Kikuchi-Fujimoto disease-Including some interesting cases. Clin Rheumatol. 2012; 31: 1463-1469. Ref.: https://goo.gl/nS27dp 
11. Fiorella ML, Gelardi M, MArzullo A, Sabattini E, Raffaele F. Kikuchi-Fujimoto disease: an uncommon cause of neck swelling. Euro Arch Oto-Rhino-Laryngol. 2017; 274: 1761-1764. Ref.: https://goo.gl/FFB8v4

12. Marunaka $\mathrm{H}$, Yorihisa $\mathrm{O}$, Tachibana $\mathrm{T}$, Kentaro $\mathrm{M}$, Makino $\mathrm{T}$, et al. Kikuchi-Fujimoto disease: evaluation of prognostic factors and analysis of pathologic findings. Acta oto-laryngol. 2016; 136. Ref.: https://goo.gl/Ax84E7

13. Brown JR, Skarin AT. Clinical mimics of lymphoma. Oncologist. 2004; 9: 406-416. Ref.: https://goo.gl/HeGNxf

14. Klotz SA, lanas V, Elliott SP. Cat-scratch Disease. Am Fam Physician. 2011; 83: 152-155. Ref.: https://goo.gl/jxS8Uq

15. Montoya JG, Liesenfeld O. Toxoplasmosis. Lancet. 2004; 363: 1965-1976. Ref.: https://goo.gl/1U2J6N 